\title{
Die Digitalisierung der Lehre und die Hochschulpolitik. Was bei der Strategiebildung nicht übersehen werden sollte
}

\section{Joachim Metzner}

\section{Zusammenfassung}

Wenn die 2020 erfolgte Umstellung der Hochschulen auf digitales Lehren und Lernen zu einer nachhaltigen positiven Veränderung der Hochschule als Bildungsinstitution beitragen soll, wird es auf eine gut durchdachte Digitalisierungsstrategie ankommen; auch eine Neujustierung bestehender Strategien kann geboten sein. Das Gleiche gilt für strategische Positionen zur Digitalisierung der Hochschulen in der europäischen und nationalen Bildungspolitik. Im Beitrag werden inhaltliche Defizite in der bisherigen Strategiebildung auf beiden Seiten aufgezeigt, die nicht zuletzt auf das Fehlen einer politischen Dimension in den hochschulischen Strategien und ein unklares Bildungsverständnis seitens der Politik zurückzuführen sind. Der Aufsatz will zu einer Aufarbeitung dieser Defizite in der aktuellen Situation anregen.

\section{Schlüsselwörter}

Strategieprozess • Hochschulpolitik • Digitalisierungsziele • Digitale

Transformation • Bildung durch Wissenschaft • Mündigkeit

\section{$1 \quad$ Einleitung}

Es herrscht in der Hochschulwelt große Einigkeit, dass der im Frühjahr 2020 plötzlich erzwungene fast vollständige Verzicht auf Präsenzlehre und -studium

\section{J. Metzner $(\varangle)$}

TH Köln, Köln, Deutschland

E-Mail: joachim.metzner@th-koeln.de 
einen massiven Bedeutungszuwachs für die Digitalisierung von Lehren, Lernen und Prüfen bringt. Die Einschätzungen der Situation reichen von der Bewertung der digitalen Lehre als vorübergehender Notlösung bis zur Prognose, die Situation werde einen wünschenswerten Durchbruch der Digitalisierung der Hochschulen auf breiter Front auslösen. Es gibt allerdings auch die gegenteilige Meinung, die Situation sei „denkbar ungünstig“ (Kerres 2020) für eine echte und sachgerechte Digitalisierung der Bildung. Dem liegt der durchaus zutreffende Eindruck zugrunde, dass unter Digitalisierung in Zeiten gesperrter Klassenräume und Hörsäle ganz überwiegend die Umstellung der herkömmlichen Lehre auf Onlinevermittlung mithilfe elektronischer Medien verstanden wird. Dabei werde - so Kerres - übersehen, dass eine sachgerechte Digitalisierung von Bildung eines strukturellen Rahmens und klar definierter Meilensteine bedarf, die auf verbindliche Ziele hin ausgerichtet sind. Die Digitalisierung von Lehren und Lernen könne nur nachhaltig gelingen, wenn sie als „komplexer integrierter Veränderungsprozess, der die gesamte Organisation betrifft“ (Kerres 2020), verstanden werde.

Die Zukunft wird zeigen, wie forcierend die Veränderungen im Jahr 2020 auf die Digitalisierung von Lehren und Lernen gewirkt haben, oder auch nicht. Aber ohne die Entwicklung einer Strategie dürfte die echte und nachhaltige Digitalisierung einer Hochschule nicht realisierbar sein. Eine plötzliche erzwungene einfache Umstellung auf online vermittelte Lehre, so schwer diese auch zu bewerkstelligen ist, kann eine Strategie nicht überflüssig machen. Da vor der Schließung der Hörsäle und Seminarräume erst gut die Hälfte aller deutschen Hochschulen über Digitalisierungsstrategien verfügte (Gilch et al. 2019, S. 65 f.), werden die übrigen eine Strategiebildung trotz umfassender Umstellung auf online vermittelte Lehre noch leisten müssen. Genauso klar ist, dass die Entwicklung im Jahr 2020 auch zur Korrektur oder Nachjustierung vieler vorhandener Strategien führen wird.

\section{Die Entwicklung der Strategiebildung und das Problem der Zielbestimmung}

Einige Hochschulen haben bereits bis 2019 hochschulische Digitalisierungsstrategien erarbeitet, sich allerdings vor allem mit der Zielbestimmung schwergetan. Im Vordergrund stand für die Hochschulen die Sicherstellung eines erfolgreichen Prozesses, was sich je nach existierender Hochschulkultur sehr unterschiedlich, aber immer schwierig gestaltete. Das gilt ebenso für die Implementierung der Strategien. Wohl deshalb ist auf eine tiefergehende Reflexion der jeweiligen Ziele 
der Digitalisierung in aller Regel weniger Gewicht gelegt worden. Die Hochschulen stimmten weitestgehend in einer instrumentellen Zielsetzung überein: Digitale Instrumente, Medien und Formate sollen zur Erleichterung oder zur Verbesserung von Lehren und Lernen nutzbar gemacht werden. Mögliche weitere Ziele kamen kaum in den Blick. Die 2020 erfolgte umfassende Umstellung der Lehre auf plötzliche Onlinevermittlung dürfte dieser Fokussierung auf eine lediglich instrumentelle Zielsetzung erneut Vorschub leisten. Doch aus dieser Einstellung ergeben sich problematische Einschränkungen und Verengungen bei hochschulischen Strategien.

Eine vollständige Sichtung der zurzeit an deutschen Hochschulen bestehenden Digitalisierungsstrategien existiert bislang nicht. Doch zeigt eine qualitative Analyse der Interessenbekundungen zur Peer-to-Peer-Strategieberatung des Hochschulforums Digitalisierung, dass den betreffenden Hochschulen das Setzen von strategischen Zielen schwerfällt und dass solche Ziele, falls sie existieren, überwiegend „selbstreferentiell“" (Watolla 2019) sind und keinen Bezug zu einem bildungs- oder hochschulpolitischen Diskurs erkennen lassen. Auch eine Untersuchung der Hochschulentwicklungspläne und Digitalisierungsstrategien von 30 staatlichen Hochschulen in NRW brachte keine Hinweise auf einen solchen Bezug (Getto und Schulenberg 2018, S. 41-44). Zugleich lassen die existierenden Digitalisierungsstrategien praktisch keine Beschäftigung mit dem im gleichen Zeitraum stattfindenden Meinungsbildungsprozess auf den unterschiedlichen politischen Ebenen erkennen, obwohl in zahlreichen Stellungnahmen und Positionspapieren unterschiedlichste Zielsetzungen formuliert und Erwartungen aus der Politik an die Hochschulen gerichtet wurden.

Ein Blick auf die Historie bestätigt und ergänzt diesen Eindruck. Die in einer ersten umfassenderen Bestandsaufnahme zur Entwicklung von Digitalisierungsstrategien an deutschen Hochschulen erhobenen Beispiele zeigten, dass nur wenige zu diesem Zeitpunkt (2016) existierende Strategien mehr sein wollten als Pläne für die Nutzung digitaler Möglichkeiten zur Verbesserung der Lehre. Wo aber das gesellschaftliche Umfeld bei der Strategieplanung eine Rolle spielte, da wurde die Digitalisierung der Lehre häufig ,,als unausweichliche Maßnahme [begriffen], um Schritt zu halten mit den technologischen Entwicklungen bzw. mit den Erwartungen der Öffentlichkeit, Politik und nicht zuletzt der heutigen Studierendengenerationen" (Schmid und Baeßler 2016, S. 9). Es ging also weniger um eine strategische Positionierung der Hochschule gegenüber externen Erwartungen, sondern oftmals um eine „Reaktion auf äußere Anreize“ (ebenda, S. 15).

In den Folgejahren hatten die Digitalisierungsstrategien zwar häufiger Profilbildung zum Ziel, nicht nur Modernisierung durch reaktive Anpassung (Getto und 
Schulenberg 2018, S. 39). Aber auch bei Strategien, die auf Profilbildung abhoben, wurden gesellschaftliche Veränderungsprozesse oder Erwartungen aus der Arbeitswelt kaum produktiv aufgegriffen. „Die Gestaltung der Lehre im digitalen Zeitalter wird an deutschen Hochschulen folglich weniger durch Entwicklungen außerhalb der Hochschulen bestimmt, sondern vielmehr durch innere Treiber gestaltet" (Schünemann und Budde 2018, S. 12). An die Politik richtete sich die Erwartung, sie möge die finanziellen Ressourcen zur Stärkung der digitalen Infrastruktur bereitstellen und die hochschulrechtlichen Regelungen an die Besonderheiten des digitalen Lehrens anpassen. Die inhaltlichen Positionen zum Thema Digitalisierung von Lehren und Lernen auf Länder- und Bundesebene wurden von den Hochschulen im Zuge der Strategiebildung wenig beachtet und noch weniger infrage gestellt.

\section{Die Erwartungen der Politik und das Verständnis von Hochschulbildung}

Dabei gab es Erwartungen der Politik an die Hochschulen, mit welchem Verständnis von Digitalisierung und mit welchen strategischen Zielsetzungen sie in eine Phase der digitalen Transformation eintreten sollten: als Positionierung der EU-Kommission, als nationale Empfehlungen, vornehmlich seitens des BMBF und der Kultusministerkonferenz, und als daraus abgeleitete Programme der Bundesländer. In diesen programmatischen Stellungnahmen und Empfehlungen wird ein sehr unterschiedliches und sich im Zeitverlauf veränderndes Verständnis von digitaler Bildung erkennbar, das den Hochschulen deshalb wenig eindeutige Orientierungshilfe bot und bietet.

\subsection{Die Erneuerungsagenda der EU-Kommission und der Aktionsplan digitale Bildung}

Seit sich EU-Gremien - etwa ab 2000 - mit Hochschulbildung für eine zunehmend digitalisierte Welt befassten, wurde Hochschulbildung ziemlich drastisch gleichgesetzt mit der Vermittlung oder Aneignung von „Skills“ zu einer den Erfordernissen des Arbeitsmarkts angemessenen Handhabung neuer Technologien. So formulierte die Europäische Kommission 2016 als Ziel, ,to enable all people to adapt to and excel in the digital economy and society" (European Commission 2016, S. 13). Eine Verlautbarung der G20-Wirtschaftsminister zeigt, wie diese Gleichsetzung sich in der internationalen Politik durchsetzte: „We also understand 
that all forms of education and life-long learning may need to be adjusted to take advantage of new digital technologies and to develop the skills required by the labour market" (G20 DEMC 2017, S. 4). Die Hochschulen in Europa wurden in einer Erneuerungsagenda aufgefordert, Lehren und Lernen systematisch zu digitalisieren, wobei unterstellt wurde, dass der Einsatz von digitalen Instrumenten und Formaten quasi automatisch zum Erwerb der für den Arbeitsmarkt notwendigen „Skills“ führt (Europäische Kommission 2017a, S. 6), eine - auch in den hochschulischen Digitalisierungsstrategien - sehr häufig zu findende, aber durchaus klärungsbedürftige Unterstellung (vgl. Aktionsrat Bildung 2018, S. 167).

Die Einstellung der EU-Kommission ist, besonders im deutschsprachigen Bereich, auf zum Teil heftige Kritik gestoßen - allerdings nicht bei bildungspolitisch Verantwortlichen oder Entscheider*innen in den Hochschulen, wohl aber in der medienwissenschaftlichen und bildungspolitischen Fachliteratur. Strategisches Ziel der sogenannten digitalen Bildung sei lediglich eine bessere Nutzung des Humankapitals. „Bildung wird als Anpassungsleistung verstanden, nicht als offener oder im gesellschaftlichen Sinne progressiver Veränderungsprozess" (Dander 2018, S. 265-267). Die Kritik ist im Ansatz berechtigt. Übersehen wird allerdings, dass die Position der EU durchaus differenzierter eingeschätzt werden kann. So betonte die Kommission an anderer Stelle, dass neben digitalen Kompetenzen der Aufbau von sogenannten Sozial- und Bürger*innenkompetenzen für ein Leben und Arbeiten im Zeitalter der Digitalität unverzichtbar sei und dass eine europäische Strategie der Digitalisierung ,mit dem Ziel der Verknüpfung von Kultur und Digitalität und der Nutzung des digitalen Potenzials zur Steigerung der positiven wirtschaftlichen und gesellschaftlichen Wirkung der Kultur“ (Europäische Kommission 2017b, S. 7; 12) verbunden sein müsse. 2018 legte die Kommission einen Aktionsplan der EU zur digitalen Bildung vor, der die Vermittlung und den Erwerb von Kompetenzen für ein Leben und Arbeiten im Zeitalter der Digitalisierung für alle Bürger*innen Europas in den Blick nahm. Der Plan bezog sich zwar auf die oben genannte Verlautbarung der Wirtschaftsminister*innen, verwies aber mit einem deutlich anderen Zungenschlag darauf, dass durch eine Mischung von sozialen und digitalen Kompetenzen ,,Resilienz in Zeiten des rasanten technologischen Wandels“ (Europäische Kommission 2018, S. 5) gestärkt werden soll. Hier zeichnet sich eine Modifikation ab, die digitale Kompetenzen zwar immer noch vornehmlich in den Dienst der wirtschaftlichen Entwicklung stellt, aber Hochschulbildung nicht auf den Erwerb digitaler Handhabungskompetenz beschränkt (Schroll et al. 2019, S. 3). Man kann Lübcke und Wannemacher zustimmen, dass seitens der EU heute ,konsequenter als in früheren Jahren auf die zur gesellschaftlichen Teilhabe befähigende Funktion von Bildung verwiesen“ (ebenda 2019, S. 35 f.) wird, dass aber diese Veränderung 
noch sehr unkonkret bleibt. Es ist noch nicht absehbar, in welche Richtung sich das Verständnis der EU-Kommission wirklich verändern wird und ob dies zu einem gewandelten Verständnis des Bildungsauftrags führt. Das für 2020 avisierte Update des Aktionsplans wird vielleicht Klarheit bringen - und hoffentlich auch zeigen, wie die EU auf die durch die Folgen der Pandemie drastisch gestiegenen Erwartungen an die Digitalisierung reagiert.

\subsection{Politische Erwartungen auf Bundesebene: Digitalisierung als nationale Aufgabe}

Die Analyse der nationalen hochschulpolitischen Aussagen und Forderungen zum Thema Bildung in der digitalen Welt macht deutlich, dass eine Neujustierung der Positionen auf Bundesebene wichtig wäre, wenn sie für die Strategiebildung der Hochschulen Relevanz bekommen sollte. Zunächst orientierten sich die Aussagen von BMBF und KMK erkennbar an der EU-Position von 2016, weichen aktuell aber in einigen wichtigen Punkten deutlich von ihr ab. Bei den neueren Positionierungen und Empfehlungen von BMBF und KMK fällt auf, dass sie von den Hochschulen zwar eine an strategischen Zielen orientierte Digitalisierung von Lehre und Studium erwarten, zu den Zielen inhaltlich aber kaum Aussagen treffen. Dies resultiert hauptsächlich aus der klaren Überzeugung, dass die durchgängige Digitalisierung aller Hochschulen längst eine ,nationale Aufgabe“ (KMK 2019, S. 4) sei, sodass das generelle strategische Ziel einer ,digitalen Transformation“ (ebenda) keiner Erörterung mehr bedürfe. 2025 soll dieses Ziel ,in einem strategiegeleiteten Verfahren" (BMBF 2019, S. 24) bei $80 \%$ aller Hochschulen erreicht sein. Bemerkenswert sind zwei weitere Argumente für den Verzicht auf Zielvorgaben: Bei der Realisierung der nationalen Aufgabe müsse die Autonomie der Hochschulen gewahrt bleiben (KMK 2019, S. 4) und die rasante Entwicklung zu einer durch umfassende Digitalität geprägten Welt mache es erforderlich, immer wieder, ,die eigenen Grundpositionen zu überdenken und daraus neue strategische Ziele abzuleiten“ (KMK 2019, S. 7). Offen bleibt, wie der Prozess einer relativ eng getakteten Überprüfung und Korrektur strategischer Ziele seitens der Hochschule gestaltet werden kann und wie sich ein solches Verfahren mit den langzeitig angelegten Ziel- und Leistungsvereinbarungen zwischen Hochschulen und Ministerien in Übereinstimmung bringen lässt.

Diese Abkehr der Politik von strategischen Vorgaben hätte einen Anstoß ergeben können, sich in den Hochschulen endlich entschiedener der Frage zuzuwenden, wie man denn nun eigentlich Digitalisierung versteht und warum und mit welchem Ziel man sie in Lehre und Studium realisieren will. Die plötzliche 
Umstellung auf Onlinelehre im Frühjahr 2020 hat diese Klärung zwar ausgesetzt, aber nur scheinbar überflüssig gemacht.

Erschwert wurde und wird den Hochschulen eine Auseinandersetzung mit politischen Positionen zur Digitalisierung der Hochschulbildung dadurch, dass BMBF und KMK ihre früheren Strategiepapiere, insbesondere die 2016 verabschiedeten, ${ }^{1}$ immer noch als richtungsweisend verstehen, auch wenn sie mit späteren Verlautbarungen nicht kompatibel sind. So wollte das BMBF 2016 mit der Bildungsoffensive für die digitale Wissensgesellschaft zwar einen „starken politischen Impuls“ (BMBF 2016, S. 15) setzen, der unter anderem die Hochschulen dazu bewegen sollte, Deutschland zu einer „Nation von Gestaltern“ (ebenda) des digitalen Wandels zu machen. Dabei wurde aber ein strategisches Tableau entwickelt, das nicht widerspruchsfrei ist. So wurde digitale Bildung verstanden als „Fähigkeit zur fachkundigen und verantwortungsvollen Nutzung digitaler Medien [...] [als] im besten Sinne integraler Bestandteil einer zeitgemäßen Allgemeinbildung" (ebenda, S. 10), orientiert an „klassischen bildungspolitischen Zielen“ (ebenda), aber Wissensvermittlung und Wissenserwerb, also typische Merkmale von klassischer Bildung, wurden ganz deutlich als nachrangig eingestuft. Hochschulen sollten sich deshalb durchaus dazu aufgerufen fühlen, für sich zu klären, in welchem Verhältnis sie digitalen Kompetenzerwerb zu ihrem generellen Auftrag Bildung durch Wissenschaft sehen. Eine Spannung besteht auch zwischen der klaren Feststellung, dass die Hochschulen autonom seien bei ihrer Strategiebildung, und dem Anspruch auf Steuerung durch die Bildungsverwaltungen: „So können die unter dem Vorzeichen der learning analytics gewonnenen Daten [von der Bildungsverwaltung] zugleich genutzt werden, um pädagogische, personalwirtschaftliche und bildungspolitische Prozesse effizienter zu steuern“ (ebenda, S. 27). Ganz ohne Zweifel hätte eine solche Steuerung Einfluss auf die strategischen Möglichkeiten der Hochschulen. Die Hochschulen sollten darauf drängen, dass Widersprüche solcher Art in politischen Strategiepapieren in Zukunft nicht mehr auftauchen.

Alle nationalen politischen Positionspapiere zur digitalen Bildung verknüpfen digitalen Kompetenzerwerb mit gesellschaftlicher Teilhabe. Digitale Bildung „soll den Menschen helfen, sich als selbstbestimmte Persönlichkeiten in einer sich beständig verändernden Gesellschaft zurechtzufinden“ (BMBF 2016, S. 4), „als selbstbestimmte Bürger aktiv an der Gesellschaft“ (KMK 2016/17, S. 17)

\footnotetext{
${ }^{1}$ Die Kultusministerkonferenz verabschiedete ihre Strategie „Bildung in der digitalen Welt“ im Dezember 2016. Die finale Fassung, die hier herangezogen wird, erschien im Dezember 2017. Sie enthält Reaktionen auf Stellungnahmen von Fachgesellschaften (vgl. Missomelius 2016, S. 6).
} 
teilzuhaben. Dass hierzu eine digitale Souveränität vonnöten ist, die neben digitaler Nutzungskompetenz eine Fülle nichtdigitaler Fähigkeiten erfordert, wurde und wird zwar regelmäßig betont, die tatsächlichen Kompetenzbeschreibungen haben jedoch einen klaren technologischen Fokus. ${ }^{2}$

$\mathrm{Zu}$ den hochschulpolitischen Strategieentwürfen auf EU- und nationaler Ebene existieren zahlreiche Reaktionen (vgl. Missomelius 2016, S. 3-5), deren generelle Tendenz sich so zusammenfassen lässt: Die Positionspapiere lassen „einen stark auf die Nutzung von digitalen Medien reduzierten Bildungsbegriff erkennen, der wesentliche Bildungserfordernisse einer durch Digitalisierung geprägten Welt leider völlig ausblendet" (Brinda 2016, S. 1). Drei Kritikpunkte, die auch Hochschulen bei ihrer Strategiebildung beachten sollten, verdienen besondere Erwähnung. So wird - neben der Kritik am einseitig auf die technologischen Kompetenzen gerichteten Fokus und der Bindung an eine ökonomistische Verwertungslogik - kritisiert, dass zwar in allen politischen Erklärungen betont wird, wie notwendig eine Kompetenz zur Gestaltung der wachsenden Digitalität aller Lebens- und Arbeitszusammenhänge ist, dass bei genauerem Hinsehen jedoch die „Gestaltungsaufgabe [...] primär in der Anpassungsleistung [besteht], die Institutionen wie Individuen an sich zu vollziehen haben" (Dander 2018, S. 271; vgl. Asmussen et al. 2018, S. 99). Deshalb sollten sich Hochschulen fragen, wie eine Hochschulstrategie der Gestaltungskompetenz - nicht nur in Hinblick auf digitale Lehr- und Lernformate, sondern auch bezogen auf eine von Digitalität geprägte Gesellschaft - trotz des „,permanenten Anpassungsdruck[s]“ (KMK 2016/17, S. 21) Vorrang einräumen kann, eines Anpassungsdrucks, der sich in Zukunft noch verstärken dürfte. Eng damit verbunden ist die Frage, ob und wie zwei der wichtigsten Ziele von Hochschulbildung im Zuge der Digitalisierung der Lehre gleichermaßen erreicht werden können: Mündigkeit durch Persönlichkeitsbildung und „employability“. Schließlich erwecken politische Stellungnahmen den Eindruck, als sei dies gerade durch Digitalisierung erreichbar, obwohl „Begriffe wie ,Beschäftigungsfähigkeit“ und ,Mündigkeit‘ [...] ganz unterschiedliche Ursprünge haben und diametral entgegengesetzte Zielvorstellungen kennzeichnen" (Aßmann 2017, S. 143). Die Hochschulen sollten sehr kritisch prüfen, wie die Vereinbarkeit solcher Zielvorstellungen - Mündigkeit als Ergebnis eines Emanzipations- und Bildungsprozesses, ,employability“ als Erwerb von berufsbezogenen Kompetenzen - unter den Vorzeichen der Digitalisierung gewährleistet werden kann (vgl. David 2019, S. 86 und öfter). Eine weitere Herausforderung ist dadurch gegeben,

\footnotetext{
${ }^{2}$ Dies dürfte darauf zurückzuführen sein, dass sich das KMK-Papier an einem sehr technologieaffinen EU-Kompetenzrahmen von 2013 orientiert (Ferrari 2013; vgl. KMK 2016/17, S. 15), während ein heute aktueller von 2018 nichtdigitale Kompetenzen betont (ESF 2018, S. 4).
} 
dass in den hochschulpolitischen - und ebenso in den meisten hochschulseitigen - Strategien der Erwerb digitaler Kompetenzen als Aufgabe und Leistung einzig von Individuen verstanden wird, was die Einbindung aller am Digitalisierungsprozess Beteiligten in eine überindividuelle ,digital citizenship“ außer Acht lässt (Macgilchrist 2019, S. 20) und manche Akteur*innenkonstellationen gar nicht wahrnimmt (Getto und Kerres 2018).

\subsection{Strategische Vorgaben auf Länderebene und Erwartungen an die Landespolitik}

Klärungsbedürftig ist auch die Rolle der Bundesländer und ihrer Wissenschaftsministerien für die Herausbildung von Hochschulstrategien. Die offizielle Erwartung der Hochschulen konzentriert sich fast unisono auf die Bereitstellung von Mitteln für den Digitalisierungsprozess und auf Gewährung eines möglichst großen Gestaltungsspielraums. Gleichwohl fällt der Landespolitik mit der Rahmengebung ein strategisches Entscheidungs- oder auch Steuerungspotenzial zu. Analysen des Hochschulforums Digitalisierung haben ergeben, dass sich die bisher, das heißt bis zur Schließung der Hörsäle im Frühjahr 2020, existierenden Digitalisierungsstrategien deutscher Hochschulen deutlich den beiden grundsätzlichen Zielsetzungen Modernisierung der Lehre oder Profilbildung für die Hochschule zuordnen lassen, wobei das Interesse an der Modernisierung deutlich überwiegt (Schünemann und Budde 2018, S. 17 f.; vgl. Getto und Schulenberg 2018, S. 39). Zumindest in Hinblick auf diese beiden strategischen Zielsetzungen der Hochschulen kann die Landespolitik durch eine programmgesteuerte Mittelvergabe mitentscheiden, „wo Modernisierungs- und wo Profilierungsthemen verfolgt werden“ (Getto und Kerres 2017, S. 134). Eine ähnliche indirekte Steuerung kann man auch der Bundespolitik zuschreiben, indem sie durch Fördermaßnahmen entweder Kooperation und damit Modernisierung oder Wettbewerb und damit Profilierung anstößt. So kann hypothetisch die Bundespolitik sogar eine strategische Einflussnahme der Länder austarieren (ebenda). Es wird sich zeigen, ob solche indirekte landes- oder bundesseitige Steuerung auch nach der Coronakrise einsetzt.

Blickt man auf die bisherige Meinungsbildung innerhalb der Hochschulen, so erhält man zur Frage der Einwirkungsmöglichkeiten der Landespolitik auf die Strategiebildung ein uneinheitliches Bild. Einerseits ist gerade die Digitalisierung kein Thema, dessen Herausforderungen und Wirkungen auf die Länder begrenzt sind. Hochschulstrategien werden also eher die Erwartungen von Fächern und länderübergreifenden ,scientific communities“ berücksichtigen als Erwartungen der Landespolitik (vgl. Getto und Kerres 2017, S. 133). Andererseits macht eine 
Umfrage unter Expert*innen für Digitalisierung der Lehre deutlich, „dass nicht die einzelnen hochschulischen Institutionen, sondern vielmehr die Bildungs- und Hochschulpolitik als Treiber für Veränderungen“ (Hochberg et al. 2019, S. 112) in den Hochschulen gesehen werden. Es existiert also innerhalb der Hochschulen eine gewisse Erwartungshaltung, dass die Politik auch auf Länderebene für den Prozess der Digitalisierung strategische Vorgaben macht. In diesem Zusammenhang ist zu erwarten, dass in Zukunft Zielvereinbarungen zwischen Ländern und Hochschulen hinsichtlich der Digitalisierung auf die 2020 gemachten Erfahrungen reagieren und eine noch wichtigere Rolle spielen werden.

Eine Analyse der einzigen repräsentativen Zusammenstellung aller länderseitigen Digitalisierungsstrategien (Arndt und Figura 2019) ergibt ein sehr differenziertes und aussagekräftiges Bild. Nicht alle Bundesländer haben eine spezielle Strategie für die Digitalisierung in der Hochschulbildung erstellt, aber alle erwarten eine zügige und auf Langfristigkeit angelegte Nutzung digitaler Instrumente und Formate in ihren Hochschulen. Die meisten strategischen Papiere lassen einen deutlichen Bezug zur KMK-Strategie von 2016/17 erkennen. Dabei wird die Steigerung von Qualität, Effektivität und Effizienz der Lehre durchgängig als Ziel oder als Erwartung formuliert (Bedenlier und Deimann 2020, S. 51). Nur in wenigen Fällen wird eine Nutzung der Digitalisierung zur Profilschärfung empfohlen. Es überwiegt der Eindruck, die Hochschulen sollten sich einem gesamtgesellschaftlichen Digitalisierungstrend anpassen. Ohne Zweifel wird dieses Drängen in Zukunft weiter zunehmen, denn auch außerhalb der Hochschulwelt werden durch die Folgen der Pandemie umfassende Digitalisierungsschübe auf zahlreiche Branchen und gesellschaftliche Institutionen zukommen.

$\mathrm{Zu}$ dieser allgemeinen Zielsetzung traten bislang landesspezifische Erwartungen, bei denen sich wiederum Schwerpunkte erkennen lassen. So erhoffen sich zahlreiche Länder von der Digitalisierung eine größere Öffnung der Hochschulen, leichtere $\mathrm{Zu}$ - und Übergänge, das Erreichen neuer Zielgruppen. An zweiter Stelle steht der Wunsch, die Hochschulen des eigenen Landes mögen durch Digitalisierung sichtbarer, wettbewerbsfähiger werden. Andererseits werden durchgängig standortübergreifende Strategien angemahnt, Digitalisierung solle Kooperation erleichtern. Unter digitaler Bildung wird überwiegend der Erwerb von instrumentellem Nutzungswissen verstanden, womit sich die Strategien der Länder in das Konzept der KMK-Strategie einfügen.

Die generellen wie auch die länderspezifischen Erwartungen fanden in zahlreichen Ziel- und Leistungsvereinbarungen mit den Hochschulen ihren Niederschlag, wobei die landesseitigen strategischen Digitalisierungsziele bislang eher den Charakter von Vorgaben hatten. Dies scheint sich aktuell im Sinne von gemeinsamer 
Zielfindung zu ändern. ${ }^{3}$ Eine Rolle spielen in diesem Zusammenhang auch die häufiger werdenden Hochschulnetzwerke zur Digitalisierung, ${ }^{4}$ die gegenüber den jeweiligen Ländern als Partner bei der strategischen Planung fungieren können.

\section{$4 \quad$ Neujustierung der Digitalisierungsstrategien als Aufgabe der Politik und der Hochschulen}

Die Situation an den deutschen Hochschulen im Jahr 2020 ist geprägt von einem extremen Bedarf an zügig und möglichst unmittelbar einsetzbaren digitalen Instrumenten, Methoden und Formaten für Lehren, Lernen und Prüfen, deren Handhabung auch Ungeübten niedrigschwellig vermittelt werden kann. Damit hat die Nutzung digitaler Möglichkeiten eine weitestgehend substitutive Funktion. Soll aus dieser Situation ein Katalysatoreffekt für eine digitale Transformation der Hochschulwelt hervorgehen (vgl. Vladova und Renz 2020), dann wird ein - gegebenenfalls erneuter - Einstieg in die Strategiebildung unabdingbar sein. Dabei werden die aktuell gemachten Erfahrungen eine prioritäre Rolle spielen. Aber die Hochschulen sollten auch die angesprochenen inhaltlichen Defizite bei der Strategiebildung möglichst angehen:

- Jede Erarbeitung einer Strategie sollte mit der Klärung des jeweiligen institutionellen Bildungsverständnisses beginnen. Dabei sollte in Zukunft die Frage im Vordergrund stehen, wie die Digitalisierung von Lehren und Lernen gestaltet werden muss, damit sie der Erfüllung des Auftrags Bildung durch Wissenschaft gerecht werden kann - nicht umgekehrt.

- Der Zielehorizont sollte nicht, wie in der Vergangenheit, auf Verbesserung oder, aktuell, auf Ermöglichung von Lehre eingeengt werden. Die Strategie sollte auf den umfassenden Veränderungsprozess von Lehren und Lernen abheben, der schon seit Jahren im Gange ist und zu dem die Digitalisierung einen forcierenden Beitrag leisten kann, wenn sie nicht mehr allein auf Krisenbewältigung fokussiert ist.

- Die Vermittlung von Kompetenzen für ein selbstbestimmtes Handeln in einer von Digitalität geprägten Lebens- und Arbeitswelt darf nicht hinter

\footnotetext{
${ }^{3}$ Der Hessische Digitalpakt Hochschulen 2020-2024 soll zurzeit in einem kooperativen strategischen Prozess entstehen. Mit „Hochschule.digital Niedersachsen“ ist eine gemeinsam mit den Hochschulen zu entwickelnde langfristig angelegte Gesamtstrategie geplant.

${ }^{4}$ Exemplarisch seien genannt: Hochschulnetzwerk Digitalisierung der Lehre BadenWürttemberg (HND-BW) sowie Netzwerk digitale Hochschullehre in Sachsen-Anhalt.
} 
der Vermittlung von Handhabungskompetenz für digitales Lehren und Lernen zurückstehen. Ein entsprechendes Kompetenzprofil sollte im Zuge der Strategiebildung erarbeitet werden.

- Jede Digitalisierungsstrategie bedarf einer (hochschul)politischen Positionierung, die den nationalen und europäischen Diskurs über Digitalisierung als gesamtgesellschaftlichen und kulturellen Transformationsprozess kritisch reflektiert und die Hochschulstrategie als Beitrag zu diesem Diskurs versteht.

Nicht nur die Hochschulen haben die Erwartung, dass die erzwungene Umstellung auf digitale Lehre im Jahr 2020 dauerhafte Effekte auf die Digitalisierung von Bildungsprozessen haben wird, auch die Politik wird sich gefordert sehen, diese Entwicklung nicht allein durch die Bereitstellung von finanziellen Mitteln zu begleiten, sondern Impulse für ein strategisches Neudenken zu setzen. Auch hier sollten die Fehler der Vergangenheit vermieden werden:

- Vor dem Hintergrund bislang unklarer und teilweise widersprüchlicher Aussagen sollte geklärt werden, was unter Hochschulbildung und Digitalisierung verstanden wird und welche Funktion politische Strategien haben sollen.

- Die politische Erwartung an die Hochschulen darf sich nicht darin erschöpfen, dass diese mithilfe von Digitalisierung den Regelbetrieb aufrechterhalten und sich stetig dem Anpassungsdruck wachsender Digitalität der Lebens- und Arbeitswelt beugen. Die Erwartung sollte vielmehr darauf gerichtet sein, dass die Hochschulen diese Digitalität innerhalb und außerhalb der Hochschulen aktiv und verantwortlich mitgestalten.

- Die Positionierungen politischer Instanzen und die daraus abgeleiteten Erwartungen sollten durchweg aus dem Dialog mit den Hochschulen heraus entwickelt werden, während diese sich ebenfalls im Strategieprozess befinden.

- Die Politik sollte bei diesem Dialog durchaus darauf achten, dass die Erwartungen, die aus der Lebens- und Arbeitswelt an die Hochschulen herangetragen werden, in deren Digitalisierungsstrategien angemessener Berücksichtigung finden, als dies in der Vergangenheit der Fall war.

\section{Literatur}

Arndt, C., \& Figura, L. (2019). Strategische Ansätze der Bundesländer zur Digitalisierung in der Hochschulbildung. Hamburg: Technische Universität Hamburg. https://doi.org/10. $15480 / 882.2060$ 
Asmussen, M., Schröder, C., \& Mardell, S. (2018). Bildung in politischen Programmen. In C. Leineweber \& C. de Witt (Hrsg.), Digitale Transformation im Diskurs. Kritische Perspektiven auf Entwicklungen und Tendenzen im Zeitalter des Digitalen (S. 97-113). Hagen: deposit_Hagen. https://ub-deposit.fernuni-hagen.de/servlets/MCRFileNodeServ let/mir_derivate_00001259/DTiD_Asmussen_Schroeder_Hardell_Bildung_politischen_ Programmen_2017.pdf. Zugegriffen: 16. Apr. 2020.

Aßmann, S. (2017). Medienbildungspolitische Positionen, Forderungen und Strategien. Bundeszentrale für politische Bildung. https://www.bpb.de/lernen/digitale-bildung/med ienpaedagogik/medienkompetenzschriftenreihe/257607/medienbildungspolitische-positi onen-forderungen-und-strategien. Zugegriffen: 16. Apr. 2020.

Bedenlier, S., \& Deimann, M. (2020). „Bildung“ und „Digitalisierung“ im Spiegel von Digitalisierungsstrategien. Zeitschrift für Hochschulentwicklung, 1, 41-59. https://doi.org/10. 3217/zfhe-15-01/03.

Brinda, T. (2016). Stellungnahme zum KMK-Strategiepapier „Bildung in der digitalen Welt“. https://bit.do/gi-iad-kmk-digbil. Zugegriffen: 17. Apr. 2020.

Bundesministerium für Bildung und Forschung [BMBF]. (2016). Bildungsoffensive für die digitale Wissensgesellschaft. Strategie des Bundesministeriums für Bildung und Forschung. Berlin: BMBF. https://www.bildung-forschung.digital/de/eine-bildungsoffe nsive-fuer-die-digitale-wissensgesellschaft-1715.html. Zugegriffen: 13. März 2020.

Bundesministerium für Bildung und Forschung [BMBF]. (2019). Digitale Zukunft: Lernen. Forschen. Wissen. Die Digitalstrategie des BMBF. Berlin: BMBF. https://www. bildung-forschung.digital/de/die-digitalstrategie-des-bmbf-2479.html. Zugegriffen: 13. März 2020.

Dander, V. (2018). Ideologische Aspekte von „Digitalisierung“. Eine Kritik des bildungspolitischen Diskurses um das KMK-Strategiepapier „Bildung in der digitalen Welt“. In C. Leineweber \& C. de Witt (Hrsg.), Digitale Transformation im Diskurs. Kritische Perspektiven auf Entwicklungen und Tendenzen im Zeitalter des Digitalen (S. 252-278). Hagen: deposit_Hagen. https://ub-deposit.fernuni-hagen.de/servlets/MCRFileNodeS ervlet/mir_derivate_00001733/DTiD_Dander_Ideologische_Aspekte_Digitalisierung_ 2018.pdf. Zugegriffen: 20. Apr. 2020.

David, L. (2019). Mündige Bürger*innen als Ziel einer kritischen Hochschullehre. In D. Jahn, A. Kenner, D. Kergel \& B. Heidkamp-Kergel (Hrsg.), Kritische Hochschullehre. Impulse für eine innovative Lehr- und Lernkultur (S. 81-96). Wiesbaden: Springer VS. https://doi. org/10.1007/978-3-658-25740-8_4.

Dolderer, M. (2019). Keine Hochschule ohne Digitalisierungsstrategie. HFD-Blog. https:// hochschulforumdigitalisierung.de/de/blog/efi-report-2019-eine-einordnung-von-vierhfd-experten. Zugegriffen: 20. Apr. 2020.

Europäische Kommission. (2017a). Mitteilung der Kommission an das Europäische Parlament, den Rat, den Europäischen Wirtschafts- und Sozialausschuss und den Ausschuss der Regionen über eine europäische Erneuerungsagenda für die Hochschulbildung, $\operatorname{COM}(2017) 247$ final. Luxemburg: Europäische Kommission.

Europäische Kommission. (2017b). Mitteilung der Kommission an das Europäische Parlament, den Rat, den Europäischen Wirtschafts- und Sozialausschuss und den Ausschuss der Regionen. Stärkung der europäischen Identität durch Bildung und Kultur, COM(2017) 673 final. Luxemburg: Europäische Kommission. 
Europäische Kommission. (2018). Mitteilung der Kommission an das Europäische Parlament, den Rat, den Europäischen Wirtschafts- und Sozialausschuss und den Ausschuss der Regionen zum Aktionsplan für digitale Bildung. COM (2018) 22 final. Luxemburg: Europäische Kommission.

European Commission. (2016). A New Skills Agenda for Europe. Working Together to Strengthen Human Capital, Employability and Competitiveness, COM(2016) 381. Final. Brüssel: Europäische Kommission.

European Social Fund [ESF] Learning, \& Skills Network. (2018). Being Digitally Competent in 2020 and Beyond: A Skills Deal for Europe! https://ec.europa.eu/esf/transnationality/ content/being-digitally-competent-2020-and-beyond-skills-deal-europe. Zugegriffen: 13. März 2020.

Ferrari, A. (2013). DIGCOMP: A Framework for Developing and Understanding Digital Competence in Europe. Luxemburg. https://www.researchgate.net/publication/282860 020_DIGCOMP_a_Framework_for_Developing_and_Understanding_Digital_Compet ence_in_Europe. Zugegriffen: 13. März 2020.

G20 Digital Economy Minister Conference [G20 DEMC] (2017). G20 Digital Economy Ministerial Declaration. Shaping Digitalisation for an Interconnected World. Düsseldorf: G20 Digital Ministers. https://www.g20.utoronto.ca/2017/170407-digitalization. html. Zugegriffen: 13. März 2020.

Getto, B., \& Schulenburg, K. (2018). Digitalisierung im Kontext strategischer Hochschulentwicklung an den Hochschulen in Nordrhein-Westfalen. In B. Getto, P. Hintze \& M. Kerres (Hrsg.), Digitalisierung und Hochschulentwicklung. Proceedings zur 26. Tagung der Gesellschaft für Medien in der Wissenschaft e. V. (S. 36-48). Münster: Waxmann. https://nbn-resolving.de/urn:nbn:de:0111-pedocs-169982. Zugegriffen: 15. Apr. 2020.

Getto, B., Hintze, P., \& Kerres, M. (2018). (Wie) Kann Digitalisierung zur Hochschulentwicklung beitragen? In B. Getto, P. Hintze \& M. Kerres (Hrsg.), Digitalisierung und Hochschulentwicklung. Proceedings zur 26. Tagung der Gesellschaft für Medien in der Wissenschaft e. V. (S. 13-25). Münster: Waxmann 2018. https://nbn-resolving.de/urn:nbn: de:0111-pedocs-169982. Zugegriffen: 15. Apr. 2020.

Getto, B., \& Kerres, M. (2017). Akteurinnen/Akteure der Digitalisierung im Hochschulsystem: Modernisierung oder Profilierung? Zeitschrift für Hochschulentwicklung, 12(1), 123-142. https://zfhe.at/index.php/zfhe/article/view/973. Zugegriffen: 03.09.2021.

Getto, B., \& Kerres, M. (2018).Wer macht was? Akteurskonstellationen in der digitalen Hochschulbildung. In B. Getto, P. Hintze \& M. Kerres (Hrsg.), Digitalisierung und Hochschulentwicklung. Proceedings zur 26. Tagung der Gesellschaft für Medien in der Wissenschaft e. V. (S. 60-73). Münster: Waxmann. https://www.pedocs.de/volltexte/2019/ 17001/pdf/MidW_74_Getto_Kerres_Wer_macht_was.pdf. Zugegriffen: 15. Apr. 2020.

Gilch, H., Beise, A. S., Krempkow, R., Müller, M., Stratmann, F., \& Wannemacher, K. (2019). Digitalisierung der Hochschulen. Ergebnisse einer Schwerpunktstudie für die Expertenkommission Forschung und Innovation. Berlin: HIS-HE. https://his-he.de/publikationen/ detail/digitalisierung-der-hochschulen. Zugegriffen: 24. Apr. 2020.

Hechler, D., \& Pasternack, P. (2017). Digitalisierungsstrategien und Digitalisierungspolicies an Hochschulen. Die hochschule. journal für wissenschaft und bildung, 2, 84-105.

Hochberg, J., Wild, R., \& Bastiaens, Th. J. (2019). Hochschulen der Zukunft. Anforderungen der Digitalisierung an Hochschulen, hochschulstrategische Prozesse und Hochschulbildungspolitik. Münster: readbox unipress. https://doi.org/10.18445/20190201-130939-0. 
Kerres, M. (2020). Digitalisierung der Bildung - Angesichts der Krise [Videoaufzeichnung]. LearningLab Universität Duisburg-Essen. Aufzeichnung vom 20.03.2020. YouTube. https://www.youtube.com/watch?v=3J1UODgp_yc. Zugegriffen: 23. Apr. 2020.

Kultusministerkonferenz [KMK]. (2017). Bildung in der digitalen Welt. Strategie der Kultusministerkonferenz. https://www.kmk.org/fileadmin/Dateien/pdf/PresseUndAktuel les/2017/Strategie_neu_2017_datum_1.pdf. Zugegriffen: 13. März 2020.

Kultusministerkonferenz [KMK]. (2019). Empfehlungen zur Digitalisierung in der Hochschullehre. Berlin, Bonn: Sekretariat der Ständigen Konferenz der Kultusminister der Länder in der Bundesrepublik Deutschland. https://www.kmk.org/fileadmin/Dateien/pdf/ PresseUndAktuelles/2019/BS_190314_Empfehlungen_Digitalisierung_Hochschullehre. pdf. Zugegriffen: 13. März 2020.

Lübcke, M., \& Wannemacher, K. (2019). Bildungsverständnis im europaweiten Vergleich. Analyse von Konzeptionen und Narrativen von EU-Kommission und ausgewählter EULänder (Arbeitspapier, 49). Berlin: Hochschulforum Digitalisierung. https://doi.org/10. 5281/zenodo.4282353.

Macgilchrist, F. (2017). Die medialen Subjekte des 21. Jahrhunderts: Digitale Kompetenzen und/oder Critical Digital Citizenship. In H. Allert, M. Asmussen \& C. Richter (Hrsg.), Digitalität und Selbst. Interdisziplinäre Perspektiven auf Subjektivierungs- und Bildungsprozesse (S. 145-168). Bielefeld: transcript.

Macgilchrist, F. (2019). Digitale Bildungsmedien im Diskurs. Wertesysteme, Wirkkraft und alternative Konzepte. Aus Politik und Zeitgeschichte, 27-28, 18-23.

Metzner, J., Bartosch, U., Vogel, M., Schroll, A., Rademacher, M., \& Neuhausen, H. (2019). Was bedeutet Hochschullehre im digitalen Zeitalter? Eine Betrachtung des Bildungsbegriffs vor den Herausforderungen der Digitalisierung (Arbeitspapier, 50). Berlin: Hochschulforum Digitalisierung. https://doi.org/10.5281/zenodo.4282368.

Missomelius, P. (2016). Die Kultusministerkonferenz und die Medienbildung - Stellungnahmen zum KMK-Strategie-Entwurf „Bildung in der digitalen Welt“. Medienimpulse, 3, S. $1-4$.

Schmid, U., \& Baeßler, B. (2016). Strategieoptionen für Hochschulen im digitalen Zeitalter (Arbeitspapier, 29). Berlin: Hochschulforum Digitalisierung. https://doi.org/10.5281/zen odo.4282185.

Schroll, A., Rademacher, M., \& Metzner, J. (2019). Zwischen „Fake News“ und „Future Skills". Was bedeuten Wahrheit, Gewissheit und Wissen in Zeiten der Digitalisierung und wie verstehen wir Bildung im 21. Jahrhundert?. Diskussionspapier, 5. Berlin: Hochschulforum Digitalisierung. https://doi.org/10.5281/zenodo.2635424.

Schünemann, I., \& Budde, J. (2018). Hochschulstrategien für die Lehre im digitalen Zeitalter: Keine Strategie wie jede andere! (Arbeitspapier, 38). Berlin: Hochschulforum Digitalisierung. https://doi.org/10.5281/zenodo.2592258.

Vladova, G., \& Renz, A. (2020). Die Coronakrise als Katalysator für die Digitalisierung von Bildungsprozessen. Ein Bericht. Berlin: Weizenbaum Institut. https://lswi.de/assets/ downloads/publikationen/110/Vladova-Die-Coronakrise-als-Katalysator-fuer-die-Digita lisierung.pdf. Zugegriffen: 20. Apr. 2020.

Von der Heyde, M., Auth, G., Hartman, A., \& Erfurth, C. (2017). Hochschulentwicklung im Kontext der Digitalisierung - Bestandsaufnahme, Perspektiven, Thesen. In M. Eibl \& M. Gaedke (Hrsg.), Informatik 2017. (S. 1757-1771). Bonn: Gesellschaft für Informatik e.V. https://doi.org/10.18420/in2017_175.

Vuorikari, R., Punie, Y., Carretero Gomez, S., \& van den Brande, G. (2016). DigComp 2.0: The Digital Competence Framework for Citizens. Update Phase 1: The Conceptual Reference 
Model. Luxemburg: Publication Office of the European Union. https://doi.org/10.2791/ 11517.

Watolla, A. (2019). Strategische Weiterentwicklung von Studium und Lehre im digitalen Zeitalter: Handlungsfelder und Herausforderungen. Diskussionspapier, 6. Berlin: Hochschulforum Digitalisierung. https://doi.org/10.5281/zenodo.3484719.

Open Access Dieses Kapitel wird unter der Creative Commons Namensnennung 4.0 International Lizenz (http://creativecommons.org/licenses/by/4.0/deed.de) veröffentlicht, welche die Nutzung, Vervielfältigung, Bearbeitung, Verbreitung und Wiedergabe in jeglichem Medium und Format erlaubt, sofern Sie den/die ursprünglichen Autor(en) und die Quelle ordnungsgemäß nennen, einen Link zur Creative Commons Lizenz beifügen und angeben, ob Änderungen vorgenommen wurden.

Die in diesem Kapitel enthaltenen Bilder und sonstiges Drittmaterial unterliegen ebenfalls der genannten Creative Commons Lizenz, sofern sich aus der Abbildungslegende nichts anderes ergibt. Sofern das betreffende Material nicht unter der genannten Creative Commons Lizenz steht und die betreffende Handlung nicht nach gesetzlichen Vorschriften erlaubt ist, ist für die oben aufgeführten Weiterverwendungen des Materials die Einwilligung des jeweiligen Rechteinhabers einzuholen.

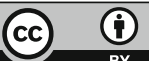

